\title{
Stress Analysis for Different Designs of Implant-borne and Tooth-implant Fixed Partial Dentures in Mandibular Posterior Region
}

\author{
Mohamed T Hamed ${ }^{1}$, Hisham A Mously ${ }^{2}$
}

\begin{abstract}
Aim: The utilization of fixed partial dentures (FPD) ceramic frameworks for replacing the missing posterior teeth is observed to have reported high failure rates. For this reason, 3-units FPD are designed and used extensively in the clinical practices, particularly for posterior mandibular region. The current study however, aims to carry out an evaluation of different stress patterns that are induced in a 3-units FPD within posterior part of mandible using finite element analysis (FEA).

Materials and methods: Different modalities of the implant-supported FPDs such as type of connector, length and diameter of implants were taken into account. A three-dimensional (3D) model simulation of a mandibular Kennedy class II removal partial denture (RPD) was designed from a computerized tomography (CT) input with digital imaging communication on medicine (DICOM) format. In addition, occlusal load of 100 $\mathrm{N}$ was also used for central fossae of first premolar, first molar and second molar of the 3-units' reconstruction for each model. The magnitude of von Mises stress including minimum stress, maximum stress and average stress were also evaluated for each loading condition.

Results: The findings of the current study showed evidence that tooth-implant design with a nonrigid connector has significantly increased the average stress. Furthermore, it was observed that rigid connector has considerably minimized the stress within the tooth-implant-supported FPD. Conclusion: The study concluded that stress designs significantly influence the stress distribution around the bone.

Clinical significance: This study gives some guidance and criteria for the selection of the type of fixed implant prosthesis in the posterior mandibular region.
\end{abstract}

Keywords: Connectors, Design modalities, Fixed prosthesis, Implants stress analysis.

The Journal of Contemporary Dental Practice (2019): 10.5005/jp-journals-10024-2707

\section{INTRODUCTION}

Premature loss of posterior teeth and lack of distal abutment are certain issues in the field of dental rehabilitation that have garnered much attention. It was noted by studies that the effective restoration of distal extension spaces is an issue that greatly plagues those individuals, who are engaged in the field of dentistry. ${ }^{1}$ In order to carry out the replacement of missing teeth, FPD with ceramic frameworks are often utilized. ${ }^{2}$ There were high failure rates reported when the posterior teeth were replaced with completely ceramic FPDs and less number of reported cases of failure related to metal-ceramic FPDs. ${ }^{3,4}$ Therefore, the use of such metal-ceramic FPDs are most commonly utilized as a replacement for posterior teeth. Additionally, the efficacy of FPDs as opposed to RPDs was also discussed. ${ }^{5}$ With this regard, 3-unit FPDs are commonly utilized for the posterior mandibular region.

The use of implant reinforced FPDs may cause stress intensification within the collateral alveolar bone. ${ }^{6}$ This development of intense stress levels may contribute to bone resorption under high occlusal loads; and thus, adversely influence the long-term efficacy of the FPDs. Therefore, it is of utmost necessity that the stress distribution which is developed over FPD should be suitably mapped and measured to determine the potential sites of maximal stress levels. The level of stress distribution within the bone is highly dependent on the positioning of the implants as well as the size and dimensions of those implants. ${ }^{7}$

Previously, a study had compared between natural tooth models and interspersed implant with rigid and nonrigid dental prostheses. ${ }^{8}$ It was found that there were minimal differences
${ }^{1}$ Department of Oral and Maxillofacial Prosthodontics, Faculty of Dentistry, King Abdulaziz University, Jeddah, Kingdom of Saud Arabia; Department of Fixed Prosthodontics, School of Dentistry, Cairo University, Al Manial, Cairo, Egypt

${ }^{2}$ Department of Oral and Maxillofacial Prosthodontics, Faculty of Dentistry, King Abdulaziz University, Jeddah, Kingdom of Saudi Arabia Corresponding Author: Mohamed T Hamed, Department of Oral and Maxillofacial Prosthodontics, Faculty of Dentistry, King Abdulaziz University, Jeddah, Kingdom of Saudi Arabia; Department of Fixed Prosthodontics, School of Dentistry, Cairo University, Al Manial, Cairo, Egypt, Phone: +966 558190492, e-mail: mohsarwat62@gmail.com

How to cite this article: Hamed MT, Mously HA. Stress Analysis for Different Designs of Implant-borne and Tooth-implant Fixed Partial Dentures in Mandibular Posterior Region. J Contemp Dent Pract 2019;20(12):1375-1379.

Source of support: Nil

Conflict of interest: None

between the magnitudes and patterns of stress initiated in the models mimicking a rigid and nonrigid dental prosthesis sustained by a natural tooth and implant. The study highlighted that the ultimate tension was in the cervical part of the implant and that the ultimate compressive stresses were exhibited in the root that supported the rigid FPDs. In studies highlighting the factors contributing to the stress generation within implants, it was seen that the loading condition was the primary cause of the stress development within the tooth-implant supported FPDs. ${ }^{9}$ 
Sharma et al. ${ }^{10}$ conducted a simulation investigation to measure the biologic performance of implants by photoelasticity and examine the stress transmission pattern with different implant supported FPDs under simulated functional loads. As indicated by the results, minimal stresses were observed at the apices of teeth or implants when forces were exerted far from the upholding abutment. Although minimal stress was the least stress marked in use of nonrigid connector, the rigid connector showed more diffuse stress transmission in the implant supported prosthesis. The use of photoelastic techniques to measure stress patterns were additionally seen in a study, which conducted a qualitative investigation of the dissemination of stress in bone supported rigid and nonrigid FPDs designs. ${ }^{11}$ It was seen that the rigid FPD design did not permit independent response by either abutment. However, the nonrigid design allowed the abutments some independence. Seemingly, the rigid restoration undertakes more stress before restoring the underlying bone.

Interpretive approaches, utilized in structural engineering, have commonly been used to assess and appraise materials and prosthetic schemes in dentistry. Therefore, the structures to be evaluated may be examined through the incorporation of stress analysis techniques. Through conducting such stress analysis under the appropriate conditions, information may be derived that is pertinent to a host of dental and clinical applications. With regard to this, the finite element method is an analytical tool for the effective stimulation of structures, through which internal stresses and force-distance relationship may be examined within those structures. $^{12}$

\section{Aim of the Study}

The study aims to carry out an evaluation of different stress patterns that are induced in a 3-units FPD within posterior part of mandible through the use of FEA. Thus, the size and length of the implants that support FPDs in the posterior mandibular region have been explored. The relative effects of different design modalities, implant length, implant diameter and rigid or nonrigid connector on stress distribution of FPDs have also been explored.

\section{Materials and Methods}

This study was conducted in King Abdulaziz University Faculty of Dentistry. A 3D finite element computer model simulation of a mandibular Kennedy class II RPD was constructed from a CT input with DICOM format processed through a Mimics software program. This study conducted an analysis of the effects of length, diameter, and connector type on the stress distribution within FPD in the posterior mandibular region. Twenty models of different designs for fixed implant prostheses were simulated and divided into two main groups. The first group is for the different designs of the 3-units implant-borne FPD based on the different combinations of implant lengths (10, 11.5 and $13 \mathrm{~mm}$ ) and implant diameter (3.5, 4.5 and 5.5 $\mathrm{mm}$ ) as variables. The second group is for the different designs of the 3-units implant-tooth FPD with regards to the mode of connection between the pontic and the tooth, whether it is a rigid or nonrigid connection. A simulated occlusal load of $100 \mathrm{~N}$ was applied over the central fossae of the first premolar, first molar and second molar of the three units' restoration for each of the 20 simulated different design models. The magnitude of von Misses stress at each element, maximum stress, minimum stress and the average stress within every part were calculated for each loading condition.
The computer simulation made use of Toshiba Astieon 4 multislice CT scanner with $140 \mathrm{kV}$ energy and 138 slices, having $512 \times 512$ resolution and pixel size $0.33 \mathrm{~mm}$. Mimics software program and Solidworks ${ }^{\circledast}$ Premium $2012 \times 64$ Edition SP 5.0 were also used in this study. This 3D model was constructed by selecting a 37-yearold volunteer with no developmental abnormalities, no gross defects, and no previous surgery to underwent a mandibular CT scanning. CT image acquisition was then performed in a DICOM format using a Toshiba multi-slice $\mathrm{CT}$ at the military production hospital. CT scanned images were exported to Mimics 8.1 program with initialization of anterior and posterior position for the axial plane and top and bottom position for other planes. This program then carried out four operations namely assembly thresholding, mandible regional growing, 3D model calculation, and exporting this 3D model to the Solidworks program. Following this, 3D drawing of the model components was carried out, namely, implant, periodontal ligament, three-unit restoration, and premolar component. Subsequently, these 3D components were assembled. It is to be noted that all the materials were considered to ideally be homogenous, isotropic, and linearly elastic. In modeling of bone both compact and cancellous, implant system, dentin, periodontal ligament and nickel-chromium FPDs, we used the modulus of elasticity and Poisson's ratio.

All the 3D components were constructed to ensure 100\% contact along each interface for removing the occurrence of any gaps. This absence of gaps between different components of the model is necessary to prevent the presence of any geometrical errors during the meshing process. Bonded contacts between two contacting surfaces along with the interface means that these objects are displayed as one unit upon load application. Structures that penetrated each other such as the bone implant, were provided with bonded type contact. The structures that did not penetrate each other were given no-slip penetration type contact. A solid mesh was planned and the resultant nodes were enabled to be translated along any of the 3 orthogonal directions unless a restraint was applied. This restraint property is a special feature in stress analysis program that allows restriction of displacements of vertices, edges, or faces for use during static analysis of the model. The restraints applied were fixed restraints on the bottom surface of mandible as well as at the condylar area of one side, i.e., no translation was allowed for these surfaces in all directions. An occlusal load of $100 \mathrm{~N}$ was applied over the central fossae of the first premolar, first molar and second molar of the three units' restoration for each of the 20 models of equal sizes. The magnitude of von Misses stress at each element, maximum stress, minimum stress and the average stress within every part were calculated for each loading condition. The study has presented increase in lengths, diameter and connectors in the form of measurement representation.

\section{Results}

The effects of length of implant, diameter of implant, and connector type on the stress distribution within FPD in the posterior mandibular region were analyzed using finite element stress analysis.

The increase in lengths and diameters for tooth-implant with rigid connection have been noted in Table 1; whereas, the increase in lengths and diameters for the tooth-implant with nonrigid connection have been noted in Table 2 . However, the results obtained for the increase in lengths and diameters 
pertaining to the implant-implant with rigid connection may be noted in Table 3; whereas, the increase in lengths and diameters noted for the implant-implant with nonrigid connections were also witnessed (Table 4). As seen in Figure 1, the highest stress value within the FPD was recorded for the tooth-implant and the nonrigid connector ( $611.267 \mathrm{MPa})$. The second highest stress within the FPD was recorded for the tooth-implant and the rigid connector

Table 1: Tooth-implant rigid connection showing the average magnitude of von Misses stresses within every part of each design for each loading condition

\begin{tabular}{lcll}
\hline & Diameter increase & \multicolumn{2}{c}{ Length increase } \\
\hline $3.5 \mathrm{~mm}$ & 6,648 & $10 \mathrm{~mm}$ & 6,648 \\
$4.5 \mathrm{~mm}$ & 5,353 & $11.5 \mathrm{~mm}$ & 5,628 \\
$5.5 \mathrm{~mm}$ & 4,046 & $13 \mathrm{~mm}$ & 4,496 \\
\hline
\end{tabular}

Table 2: Tooth-implant nonrigid connection showing the average magnitude of von Misses stresses within every part of each design for each loading condition

\begin{tabular}{llll}
\hline & Diameter increase & \multicolumn{2}{c}{ Length increase } \\
\hline $3.5 \mathrm{~mm}$ & 5,790 & $10 \mathrm{~mm}$ & 5,790 \\
$4.5 \mathrm{~mm}$ & 4,982 & $11.5 \mathrm{~mm}$ & 5,120 \\
$5.5 \mathrm{~mm}$ & 3,783 & $13 \mathrm{~mm}$ & 4,131 \\
\hline
\end{tabular}

Table 3: Implant-implant rigid connection showing the average magnitude of von Misses stresses within every part of each design for each loading condition

\begin{tabular}{llll}
\hline & Diameter increase & \multicolumn{2}{c}{ Length increase } \\
\hline $3.5 \mathrm{~mm}$ & 5,409 & $10 \mathrm{~mm}$ & 5,409 \\
$4.5 \mathrm{~mm}$ & 4,801 & $11.5 \mathrm{~mm}$ & 4,940 \\
$5.5 \mathrm{~mm}$ & 3,461 & $13 \mathrm{~mm}$ & 4,278 \\
\hline
\end{tabular}

Table 4: Implant-implant nonrigid connection showing the average magnitude of von Misses stresses within every part of each design for each loading condition

\begin{tabular}{lcll}
\hline & Diameter increase & \multicolumn{2}{c}{ Length increase } \\
\hline $3.5 \mathrm{~mm}$ & 4,917 & $10 \mathrm{~mm}$ & 4,917 \\
$4.5 \mathrm{~mm}$ & 4,247 & $11.5 \mathrm{~mm}$ & 4,460 \\
$5.5 \mathrm{~mm}$ & 3,299 & $13 \mathrm{~mm}$ & 3,481 \\
\hline
\end{tabular}

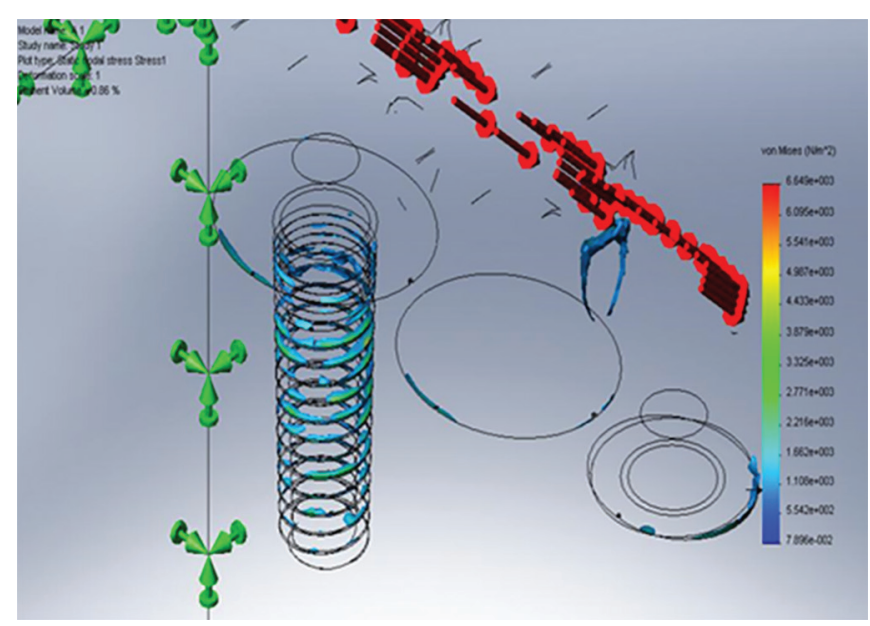

Fig. 1: Effect of diameter on stresses
(388.6 MPa), followed by implant-implant supporting FPD and the nonrigid connector (113.284 MPa), with an $81.4 \%$ decrease in stress from the highest value. Finally, the lowest stress value within FPD was recorded for the implant-implant with the rigid connector (96.168 MPa), which showed an $84.2 \%$ decrease in stress from the highest value.

The tooth-implant design with a nonrigid connector showed the significantly highest mean stress. It was followed by the toothimplant design with the rigid connector and the implant-implant design with the nonrigid connector. It was seen that the implantimplant design with the rigid connector showed the lowest stress. Furthermore, with either the tooth-implant or implant-implant supported FPD, it was seen that the nonrigid connector showed significantly higher stresses than the rigid connector did. These results have been tabulated in Tables 5 and 6 .

It was further seen that the rigid connector reduced the stress induced in the FPD by $36.4 \%$ within the tooth-implant supported FPD design. This stress reduction by the rigid connector was noted to be at a level of $15.1 \%$ within the implant-implant supported FPD design. The effect of diameters on the stress of FPDs have been noted in Table 7 and Figure 1. Furthermore, the effect of lengths on the stress of the FPDs have been noted in Table 8 .

\section{Discussion}

In the current study, it has been observed that implant designs with nonrigid connector indicate considerably higher stress mean. On

Table 5: Tooth-implant rigid vs nonrigid connection showing the average magnitude of von Misses stresses within every part of each design for each loading condition

\begin{tabular}{lll}
\hline & Rigid connection & Nonrigid connection \\
\hline $\begin{array}{l}\text { Standard implant } 3.5 \mathrm{~mm} \\
\text { diameter and } 10 \mathrm{~mm}\end{array}$ & 6,648 & 5,790 \\
$\begin{array}{l}\text { length } \\
\text { Diameter increased }\end{array}$ & 5,353 & 4,982 \\
$\begin{array}{l}4.5 \mathrm{~mm} \text { and } 10 \mathrm{~mm} \text { length } \\
\text { Diameter increase } 5.5 \mathrm{~mm} \\
\text { and } 10 \mathrm{~mm} \text { length }\end{array}$ & 4,046 & 3,783 \\
$\begin{array}{l}\text { Length increased to } \\
11.5 \mathrm{~mm} \text { and } 3.5 \mathrm{~mm} \\
\text { diameter }\end{array}$ & 5,628 & 5,120 \\
$\begin{array}{l}\text { Length increased to } \\
13 \mathrm{~mm} \text { and } 3.5 \mathrm{~mm} \\
\text { diameter }\end{array}$ & 4,496 & \\
\hline
\end{tabular}

Table 6: Implant-implant rigid vs nonrigid connection

\begin{tabular}{lll}
\hline & Rigid connection & Nonrigid connection \\
\hline $\begin{array}{l}\text { Standard implant } 3.5 \mathrm{~mm} \\
\text { diameter and } 10 \mathrm{~mm}\end{array}$ & 5,409 & 4,917 \\
length & & \\
$\begin{array}{l}\text { Diameter increased } \\
4.5 \mathrm{~mm} \text { and } 10 \mathrm{~mm} \text { length }\end{array}$ & 4,801 & 4,247 \\
$\begin{array}{l}\text { Diameter increase } 5.5 \mathrm{~mm} \\
\text { and } 10 \mathrm{~mm} \text { length }\end{array}$ & 3,461 & 3,299 \\
$\begin{array}{l}\text { Length increased to } \\
11.5 \mathrm{~mm} \text { and } 3.5 \mathrm{~mm} \\
\text { diameter } \\
\text { Length increased to }\end{array}$ & 4,940 & 4,460 \\
$\begin{array}{l}13 \mathrm{~mm} \text { and } 3.5 \mathrm{~mm} \\
\text { diameter }\end{array}$ & 4,278 & \\
\hline
\end{tabular}


Table 7: Effect of diameter increase on stresses

\begin{tabular}{lll}
\hline & Medium implant & \\
Thin implant $3.5 \mathrm{~mm}$ & $4.5 \mathrm{~mm}$ & Thick implant $5.5 \mathrm{~mm}$ \\
\hline 6,648 & 5,353 & 4,046 \\
5,790 & 4,982 & 3,783 \\
5,409 & 4,801 & 3,461 \\
4,917 & 4,247 & 3,299 \\
\hline
\end{tabular}

Table 8: Effect of length increase on stresses

\begin{tabular}{lll}
\hline & Medium implant & \\
Short implant 10 mm & $11.5 \mathrm{~mm}$ & Long implant 13 $\mathrm{mm}$ \\
\hline 6,648 & 5,628 & 4,496 \\
5,790 & 5,120 & 4,131 \\
5,409 & 4,940 & 4,278 \\
4,917 & 4,460 & 3,481 \\
\hline
\end{tabular}

the contrary, implant designs with rigid connector decreased the stress. Furthermore, the current study also indicated that increase in the length of implants leads to an increase in the stress distribution. Increase in diameter of implants is directly proportional to the decrease in stress. The two distinct methods that are used to study the biomechanical patterns of dental implants include FEA and prostate-specific antigen (PSA). The major difference between these two significant methods is the isolation of stress concentrations. On one hand, the stress concentration is determined to be higher around the implant neck in the FEA studies whereas, in the PSA studies stress concentrations are higher at the apex of implant.

Cicciù et al. ${ }^{13}$ have argued that it is difficult to measure the stress distribution around the dental implants by using sensors as well as it is also difficult to measure their quality and quantity inside the bone. Furthermore, Pessoa et al. ${ }^{14}$ have conducted in vitro procedures for observing the biomechanics of stress distribution in dentistry. Moreover, PSA has found to possess some limitations such as the resins that are applied for activating the bone, which is homogenous and has isotropic features, while the bone is found to be anisotropic. In addition, the photoelastic resin is found to increase the stresses inside the bone. Therefore, the current study has constructed a 3D model simulation of a mandibular Kennedy class II RPD and adopted FEA approach to understand the behavior of implants with FPD and bone under stresses.

da Costa Valente et al. ${ }^{15}$ have argued that this condition is determined to be independent from the type of implant utilized or the implant abutment connection. Similarly, Jensen et al. ${ }^{16}$ have indicated significant differences in a homogeneous photoelastic model based on the features of implant surface as well as transformations in the implant designs.

Yang et al. ${ }^{17}$ had selected different loading points. The most common loading points that were selected in the previous studies include central fossa and functional cusps. It is however, essential to insert the tooth-implants under the central fossa to achieve ideal loading of implants utilized for posterior teeth. According to Rodrigues et al., ${ }^{18}$ the inclined implants and straight placed implants indicate non-symmetrical and symmetrical fringe patterns under loading situations, respectively. Vertical loading at the inclined implants is observed to be an oblique loading that is not parallel to implant axis thus, causing fringe patterns and non-symmetrical stress distribution around the implant apex.

Tribst et al. ${ }^{19}$ have determined the survival for a longer period in contrast to straight implants. Mandibular molars are observed to have inclined at $10^{\circ}$ mesially and higher stress values around the straight implants are greater than $10^{\circ}$. According to de Souza Batista et al., ${ }^{20}$ an inclined implant is considered to possess higher surface area to assist occlusal plane. In the current study, rigid and inclined implants reduced the stresses as compared to nonrigid or straight placed implants. The restriction of the stress concentrations could be the causative factor of bone resorption.

Several implant designs have been constructed in the dental market. Gümrükçü et al. ${ }^{21}$ have indicated that the implant design possesses a high impact on the stabilization of primer. The implants with increased diameter and length tend to have better stress distribution. In addition, Minatel et al. ${ }^{22}$ have reported that further increase in the diameter of implants could further increase stresses thus, reducing bone support. Thus, the clinicians are able to utilize optimum implant for enhanced placement. The diameter and length of the implants used in the current study to some extent are like the length and diameter of optimum implants. Certain previous studies have also stated that the implants with rigid connector, non-tapered, increased length and diameter possess better stress distribution aspects. Sommers et al. ${ }^{23}$ have found that the stepped implant types possess better stress distribution.

This study has a limitation of being a computer model simulation and approximations of the fixed implant prosthesis in the oral cavity which is not a close representation of the real functional loads and environmental factors that occur in the oral cavity. That involved several approximations in the material properties of the tissues. All teeth were given the mechanical properties of dentin. The homogenous, isotropic, and linear elastic assumption described for all the materials in the study does not apply practically to the bone which shows heterogeneous, anisotropic, and viscoelastic nature. Fixed partial dentures were used in the current study suggesting that it must be used in the modern practices as it supports the meshing between adjoining solid surfaces. Moreover, the difference between principal stress and von Mises stress was also determined during the examination of stress distribution around bone.

\section{Conclusion}

The current study has used 3D models for evaluating the impact of different lengths and diameter of implants on the stress distribution around mandibular region. According to the findings of the study, it is concluded that an increase in the implant diameter is directly proportional to reduced stress. In addition, a moderate increase in implant's length decreases the stress dissemination around bone. On the other hand, greater expansion in the length of implant ultimately increases the stress distribution. Therefore, it is concluded that different lengths and diameters of implants have different impact on the stress distribution. However, the tooth-implant models succeeded in minimizing the stress around premolar.

The current study suggested that bone is a fragile material and appropriate principal stress designs must be considered. In addition, it is also concluded that implant designs with nonrigid connector rather than rigid connector significantly increase the average stress distribution. 


\section{References}

1. Kukunoor S, Savadi RC, Raju KV, et al. A viable treatment alternative in distal extension cases: a case report. J Indian Prosthodont Soc 2014;14(Suppl 1):177-180. DOI: 10.1007/s13191-012-0218-6.

2. Raigrodski AJ, Chiche GJ. All-ceramic fixed partial dentures, part I: in vitro studies. J Esthet Restor Dent 2002;14(3):188-191. DOI: 10.1111/j.1708-8240.2002.tb00518.x.

3. Olsson KG, Fürst B, Andersson B, et al. A long-term retrospective and clinical follow-up study of in-ceram alumina FPDs. Int J Prosthodont 2003;16(2):150-156. DOI: 10.1016/s0022-3913(03)00475-x.

4. Scurria MS, Bader JD, Shugars DA. Meta-analysis of fixed partial denture survival: prostheses and abutments. J Prosthet Dent 1998;79(4):459-464. DOI: 10.1016/s0022-3913(98)70162-3.

5. Yi SW, Carlsson GE, Ericsson I, et al. Patient evaluation of treatment with fixed implant-supported partial dentures. J Oral Rehabil 2001;28(11):998-1002. DOI: 10.1046/j.1365-2842.2001.00819.x.

6. Stegaroiu R, Sato T, Kusakari $H$, et al. Influence of restoration type on stress distribution in bone around implants: a three-dimensional finite element analysis. Int J Oral Maxillofac Implants 1998;13(1):82-90.

7. Pilliar RM, Deporter DA, Watson PA, et al. Dental implant design-effect on bone remodeling. J Biomed Mater Res 1991;25(4):467-483. DOI: 10.1002/jbm.820250405.

8. Tsouknidas A, Giannopoulos D, Savvakis S, et al. The influence of bone quality on the biomechanical behavior of a tooth-implant fixed partial denture: a three-dimensional finite element analysis. Int J Oral Maxillofac Implants 2016;31(6):e143-e154. DOI: 10.11607/jomi.5254.

9. Lin $\mathrm{CL}$, Wang JC, Chang WJ. Biomechanical interactions in toothimplant-supported fixed partial dentures with variations in the number of splinted teeth and connector type: a finite element analysis. Clin Oral Implant Res 2008;19(1):107-117. DOI: 10.1111/j.16000501.2007.01363.x.

10. Sharma A, Mehta S, Chopra A, et al. Tooth-implant connection: a review. World Journal of Pharmacy and Pharmaceutical Sciences 2017;6(11):405-416.

11. Modi R, Kohli S, Rajeshwari K, et al. A three-dimension finite element analysis to evaluate the stress distribution in tooth supported 5-unit intermediate abutment prosthesis with rigid and nonrigid connector. Eur J Dent 2015;9(2):255. DOI: 10.4103/1305-7456.156847.

12. Yokoyama S, Wakabayashi N, Shiota M, et al. The influence of implant location and length on stress distribution for three-unit implantsupported posterior cantilever fixed partial dentures. J Prosthet Dent 2004;91(3):234-240. DOI: 10.1016/j.prosdent.2003.12.017.
13. Cicciù $M$, Cervino $G$, Milone $D$, et al. FEM investigation of the stress distribution over mandibular bone due to screwed overdenture positioned on dental implants. Materials (Basel) 2018;11(9):1512. DOI: 10.3390/ma11091512.

14. Pessoa RS, Sousa RM, Pereira LM, et al. Bone remodeling around implants with external hexagon and morse-taper connections: a randomized, controlled, split-mouth, clinical trial. Clin Implant Dent Relat Res 2017;19(1):97-110. DOI: 10.1111/cid.12437.

15. da Costa Valente ML, de Castro DT, Macedo AP, et al. Comparative analysis of stress in a new proposal of dental implants. Mater Sci Eng CMater Biol Appl 2017;77:360-365. DOI: 10.1016/j.msec.2017.03.268.

16. Jensen C, Meijer HJ, Raghoebar GM, et al. Implant-supported removable partial dentures in the mandible: a 3-16 year retrospective study. J Prosthodont Res 2017;61(2):98-105. DOI: 10.1016/j. jpor.2016.07.002.

17. Yang $\mathrm{H}$, Park C, Shin JH, et al. Stress distribution in premolars restored with inlays or onlays: 3D finite element analysis. J Adv Prosthodont 2018;10(3):184-190. DOI: 10.4047/jap.2018.10.3.184.

18. Rodrigues VA, Tribst JP, de Santis LR, et al. Influence of angulation and vertical misfit in the evaluation of micro-deformations around implants. Br Dent Sci 2017;20(1):32-39. DOI: 10.14295/bds.2017. v20i1.1311.

19. Tribst JP, Rodrigues VA, Dal Piva AO, et al. The importance of correct implants positioning and masticatory load direction on a fixed prosthesis. J Clin Exp Dent 2018;10(1):e81-e87. DOI: 10.4317/ jced.54489.

20. de Souza Batista VE, Verri FR, de Faria Almeida DA, et al. Evaluation of the effect of an offset implant configuration in the posterior maxilla with external hexagon implant platform: a 3-dimensional finite element analysis. J Prosthet Dent 2017;118(3):363-371. DOI: 10.1016/j. prosdent.2016.10.033.

21. Gümrükçü Z, Korkmaz YT, Korkmaz FM. Biomechanical evaluation of implant-supported prosthesis with various tilting implant angles and bone types in atrophic maxilla: a finite element study. Comput Biol Med 2017;86:47-54. DOI: 10.1016/j.compbiomed.2017.04.015.

22. Minatel L, Verri FR, Kudo GA, et al. Effect of different types of prosthetic platforms on stress-distribution in dental implantsupported prostheses. Mater Sci Eng C Mater Biol Appl 2017;71:35-42. DOI: 10.1016/j.msec.2016.09.062.

23. Sommers MB, Sanders RW, Falkner JG, et al. System and method for bone fixation using a nail locked to an encircling anchor. US Pat 16/153,460, 2019. 